VOL. 63 (2001) [379-391]

\title{
MORE CRANKS AND $t$-CORES
}

\author{
F.G. GARVAN
}

\section{Dedicated to George Szekeres on the occasion of his 90th Birthday}

In 1990, new statistics on partitions (called cranks) were found which combinatorially prove Ramanujan's congruences for the partition function modulo 5, 7, 11 and 25 . The methods are extended to find cranks for Ramanujan's partition congruence modulo 49. A more explicit form of the crank is given for the modulo 25 congruence.

\section{INTRODUCTION}

Let $p(n)$ be the number of partitions of $n$ [1]. If $\alpha \geqslant 1$, and $\delta_{\alpha}, \lambda_{\alpha}, \mu_{\alpha}$ are the reciprocals of 24 modulo $5^{\alpha}, 7^{\alpha}, 11^{\alpha}$ respectively, then

$$
\begin{aligned}
p\left(5^{\alpha} n+\delta_{\alpha}\right) \equiv 0 & \left(\bmod 5^{\alpha}\right), \\
p\left(7^{2 \alpha-1} n+\lambda_{2 \alpha-1}\right) \equiv 0 & \left(\bmod 7^{\alpha}\right), \\
p\left(7^{2 \alpha} n+\lambda_{2 \alpha}\right) \equiv 0 & \left(\bmod 7^{\alpha+1}\right), \\
p\left(11^{\alpha} n+\mu_{\alpha}\right) \equiv 0 & \left(\bmod 11^{\alpha}\right) .
\end{aligned}
$$

These are Ramanujan's partition congruences. Watson [9] proved (1.1), (1.2), (1.3) and Atkin [3] proved (1.4). Dyson [5] was the first to consider explaining these congruences combinatorially. Dyson defined an integral statistic on partitions, called the rank, whose value mod 5 he conjectured split the partitions of $5 n+4$ into 5 equal classes, thus giving a combinatorial refinement for the $\alpha=1$ case of (1.1). He further conjectured that the analogous result for the rank mod 7 gave the $\alpha=1$ case of (1.2), and that there was a statistic, called the crank, which would similarly give the $\alpha=1$ case of (1.4). Atkin and Swinnerton-Dyer [4] proved Dyson's rank conjecture for 5 and 7. Andrews and Garvan [2] proved Dyson's crank conjecture by finding a crank which proves not only Ramanujan's conjecture for 11 but also for 5 and 7. Later, Garvan, Kim and Stanton [6] found new cranks which gave new interpretations of Ramanujan's congruences mod 5, 7, 11, and 25 . Their approach was combinatorial and in terms of the $t$-core of a partition. They gave explicit bijections between the equinumerous classes. In the present paper we extend the

Received 5th December, 2000

Research supported in part by the NSF under grant numbers DMS-9208813 and DMS-9870052.

Copyright Clearance Centre, Inc. Serial-fee code: 0004-9727/01 \$A2.00+0.00. 
methods of [6] and give a crank which is a combinatorial refinement of the $\alpha=1$ case of (1.3), namely

$$
p(49 n+47) \equiv 0 \quad(\bmod 49) .
$$

In Section 2 we re-examine two bijections from [6]. A crank for the partitions of $25 n+24 \bmod 25$ was given in [6]. A more explicit form of this crank is given in Theorem 3.4. A new and explicit crank for the 7 -cores of $49 n+47$ is given in Theorem 3.5. This leads to a crank for the partitions of $49 n+47$ (Corollary 3.1).

\section{TWO BIJECTIONS FOR $t$-CORES}

We need to examine in detail the two bijections relating partitions and $t$-cores which were given in [6]. Following [6] we let $P$ be the set of all partitions. For any $\lambda \in P$, let $|\lambda|$ denote the number that $\lambda$ partitions. Fix a positive integer $t$. Let $P_{t \text {-core }}$ be the set of partitions which are $t$-cores. Recall that a partition is a $t$-core if it has no hook numbers that are multiples of $t$ or equivalently no rim hooks that are multiples of $t$. See [7] for background on $t$-cores, hook numbers and rim hooks. We let $a_{t}(n)$ denote the number of partitions of $n$ which are $t$-cores.

BiJection 1. $([7,2.7 .17],[6, \mathrm{p} .2]$.$) \quad There is a bijection \phi_{1}: P \rightarrow P_{t \text {-core }} \times P \times \cdots \times P$,

$$
\phi_{1}(\lambda)=\left(\tilde{\lambda}, \hat{\lambda}_{0}, \hat{\lambda}_{1}, \ldots, \hat{\lambda}_{t-1}\right),
$$

such that

$$
|\lambda|=|\widetilde{\lambda}|+t \sum_{i=0}^{t-1}\left|\widehat{\lambda}_{i}\right|
$$

Corollary 2.1.

$$
\sum_{n \geqslant 0} a_{t}(n) q^{n}=\prod_{n=1}^{\infty} \frac{\left(1-q^{t n}\right)^{t}}{\left(1-q^{n}\right)} .
$$

Given a partition $\lambda$ we label a cell in the $i$-th row and $j$-th column by $j-i(\bmod t)$. The resulting diagram is called a $t$-residue diagram [7, p.84]. We form the extended $t$-residue diagram by adding an infinite column 0 labelled in the same way. A region $r$ of the extended diagram is the set of cells $(i, j)$ with $t(r-1) \leqslant j-i<t r$. A cell is exposed if it is at the end of a row. The partition $\lambda$ is a $t$-core if and only if for each exposed cell labeled $i$ in region $r$ there is an exposed cell labeled $i$ in each region $<r$. Now we construct $t$ bi-infinite words $W_{0}, W_{1}, \ldots, W_{t-1}$ of two letters $N$ (not exposed) and $E$ (exposed):

$$
\text { The } j \text {-th element of } W_{i}= \begin{cases}N & \text { if } i \text { is not exposed in region } j, \\ E & \text { if } i \text { is exposed in region } j .\end{cases}
$$

We now give the bijection. For each $i$ we do the following steps: 
Step 1. Find the right-most $E$.

Step 2. Find the right-most $N$ to the left of this $E$. If no such $N$ exists then END.

Step 3. Remove the rim hook whose head is at $E$ and whose tail is one cell to the right of the $N$. Place a part of size (rim hook removed) $/ t$ in $\lambda_{i}$.

Step 4. Go to Step 1.

The operation in Step 3 above changes a substring of $W_{i}$ of the form $N E E \ldots E E N$ to $E E E \ldots E N N$, that is, the $N$ is pushed right.The other words $W_{j}$ are left unchanged by removing this rim hook, and we can process the $i$ 's in any order. Steps 1-4 create a partition $\lambda_{i}$ starting from the smallest part to the largest part and the process is easily reversible. At the end when all the $W_{i}$ have been processed we are left with the required $t$-core $\tilde{\lambda}$.

BiJection 2. [6, p.3] There is a bijection $\phi_{2}: P_{t \text {-core }} \rightarrow\left\{\vec{n}=\left(n_{0}, n_{1}, \ldots, n_{t-1}\right): n_{i} \in\right.$ $\left.\mathbb{Z}, n_{0}+\cdots+n_{t-1}\right\}$, where

$$
|\tilde{\lambda}|=t\|\vec{n}\|^{2} / 2+\vec{b} \cdot \vec{n}, \quad \vec{b}=(0,1, \ldots, t-1) .
$$

For a partition $\lambda$, we let $r_{k}(\lambda)$ denote the number of cells in the $t$-residue diagram labeled $k(\bmod t)$, and call

$$
\vec{r}=\left(r_{0}, r_{1}, \ldots, r_{t-1}\right)
$$

the $r$-vector of $\lambda$. Bijection 2 is given by

$$
\phi_{2}(\tilde{\lambda})=\vec{n}=\left(r_{0}-r_{1}, r_{1}-r_{2}, \ldots, r_{t-1}-r_{0}\right)
$$

Let $[x]$ denote the greatest integer not exceeding $x$. We shall need the following

LEMMA 2.1. Let $\lambda: \lambda_{1} \geqslant \lambda_{2} \geqslant \cdots \geqslant \lambda_{m}$ be a partition and suppose

$$
\phi_{1}(\lambda)=\left(\tilde{\lambda}, \hat{\lambda}_{0}, \widehat{\lambda}_{1}, \ldots, \widehat{\lambda}_{t-1}\right)
$$

Then

$$
\sum_{i=0}^{t-1}\left|\widehat{\lambda}_{i}\right|=r_{0}-\left(\sum_{i=0}^{t-1} r_{i}^{2}-r_{i} r_{i+1}\right)
$$

and

$$
\sum_{i=0}^{t-1} i\left|\widehat{\lambda}_{i}\right| \equiv \sum_{j=1}^{m}\left(\lambda_{j}-j\right)\left[\frac{\lambda_{j}-j}{t}\right]-\sum_{i=1}^{t-1} i d_{i}\left(\frac{1}{2}\left(d_{i}+1\right)+\left[\frac{m-i-1}{t}\right]\right)(\bmod t)
$$

where $d_{i}$ is the number of elements of the sequence

$$
\lambda_{1}-1, \lambda_{2}-2, \ldots, \lambda_{m}-m
$$

which are congruent to $i(\bmod t)$. 
Proof: For $t$-cores, we have

$$
r_{0}=\sum_{i=0}^{t-1}\left(r_{i}^{2}-r_{i} r_{i+1}\right)
$$

See [6, p.6]. Now suppose $\vec{r}$ is the $r$-vector of $\lambda$ and $\overrightarrow{r^{\prime}}$ is the $r$-vector of its $t$-core $\tilde{\lambda}$. The partition $\tilde{\lambda}$ is obtained from $\lambda$ by the removal of rim hooks whose lengths are multiples of $t$. Each rim hook of length $t$ contains cells with distinct $t$-residues. It follows that

$$
r_{i}^{\prime}+s=r_{i}
$$

where

$$
s=\sum_{j=0}^{t-1}\left|\widehat{\lambda}_{j}\right| .
$$

Since $\overrightarrow{r^{\prime}}$ is the $r$-vector of a $t$-core we have

$$
\begin{aligned}
r_{0}^{\prime} & =\sum_{i=0}^{t-1}\left({r^{\prime}}_{i}^{2}-{r^{\prime}}^{\prime} r^{\prime}{ }_{i+1}\right), \\
r_{0}-s & =\sum_{i=0}^{t-1}\left(\left(r_{i}-s\right)^{2}-\left(r_{i}-s\right)\left(r_{i+1}-s\right)\right) \\
& =\sum_{i=0}^{t-1}\left(r_{i}^{2}-r_{i} r_{i+1}\right),
\end{aligned}
$$

and (2.2) follows.

We add $t$ dummy zeros to the parts of $\lambda$ :

$$
\lambda_{1} \geqslant \lambda_{2} \geqslant \cdots \geqslant \lambda_{m} \geqslant 0 \geqslant \cdots \geqslant 0,
$$

and form the sequence

$$
\bar{\lambda}: \lambda_{1}-1>\lambda_{2}-2>\cdots>\lambda_{m}-m>-m-1>\cdots>-m-t .
$$

Let

$$
\overline{\mu_{i}}: \mu_{i, 1}>\mu_{i, 2}>\cdots>\mu_{i, k_{i}}
$$

be the terms of the sequence $\bar{\lambda}$ that are congruent to $i(\bmod t)$. Here $k_{i}$ is the number of terms so that $d_{i}=k_{i}-1$. Each $\mu_{i, k}$ corresponds to an exposed cell labeled $i$ in region $\left[\mu_{i, k} / t\right]+1$. In Bijection 1, the numbers

$$
n_{i, k}=\left[\frac{\mu_{i, k}}{t}\right]-\left[\frac{\mu_{i, k+1}}{t}\right]-1
$$

correspond to a string of $n_{i, k}$ consecutive $N$ 's in the word $W_{i}$. Since these $N$ 's are shifted as far as possible to the right we find that the sum of parts of the $(i+1)$ th component

$$
\left|\widehat{\lambda}_{i}\right|=n_{i, 1}+2 n_{i, 2}+\cdots\left(k_{i}-1\right) n_{i, k-1}
$$




$$
=\left[\frac{\mu_{i, 1}}{t}\right]+\cdots+\left[\frac{\mu_{i, k_{i}-1}}{t}\right]-\frac{1}{2} k_{i}\left(k_{i}-1\right)-\left(k_{i}-1\right)\left[\frac{\mu_{i, k_{i}}}{t}\right] .
$$

Since $\mu_{i, k} \equiv i(\bmod t)$ we find that

$$
\sum_{i=0}^{t-1} i\left(\left[\frac{\mu_{i, 1}}{t}\right]+\cdots+\left[\frac{\mu_{i, k_{i}-1}}{t}\right]\right) \equiv \sum_{j=1}^{m}\left(\lambda_{j}-j\right)\left[\frac{\lambda_{j}-j}{t}\right](\bmod t) .
$$

The desired result (2.3) follows from the fact that $d_{i}=k_{i}-1$ and that

$$
\left[\frac{\mu_{i, k_{i}}}{t}\right]=\left[\frac{m-i-1}{t}\right] \text {. }
$$

\section{CRANKS FOR $t$-CORES AND PARTITIONS}

We need the crank results in [6]. The following theorem follows from [6, Theorem 1].

ThEOREM 3.1. [6] If $(t, \delta)=(5,4),(7,5)$ or $(11,6)$, then

$$
\sum_{n \geqslant 0} a_{t}(t n+\delta) q^{n+1}=\sum_{\vec{\alpha} \in \mathbf{Z}^{t}, \vec{\alpha} \cdot \bar{I}=1} q^{Q(\vec{\alpha})}
$$

where

$$
Q(\vec{\alpha})=\|\vec{\alpha}\|^{2}-\sum_{i=0}^{t-1} \alpha_{i} \alpha_{i+1} .
$$

The form $Q(\vec{\alpha})$ remains invariant under a cyclic permutation of the $\alpha_{i}$. This induces a $t$-cycle on $t$-cores of $t n+\delta$, which in turn induces a $t$-cycle on partitions of $t n+\delta$ via Bijection 1. For the form $Q(\vec{\alpha})$ the associated crank statistic is $\sum_{i=0}^{t-1} i \alpha_{i}$. This leads to crank statistics for $t$-cores of $t n+\delta$, and for partitions of $t n+\delta$.

\subsection{CRANKS FOR PaRTITIONS OF $5 n+4$ AND $25 n+24$}

THEOREM 3.2. [6, p.7] Let $\vec{r}=\left(r_{0}, r_{1}, \ldots, r_{6}\right)$ be the $r$-vector of $\lambda$, a 5-core of $5 n+4$. Then

$$
c_{1}(\lambda):=2 r_{1}-r_{2}+r_{3}-2 r_{4} \quad(\bmod 5) \in \mathbb{Z}_{5}
$$

is a crank for 5 -cores of $5 n+4$.

We make explicit the 5-cycle $\sigma$ that acts on 5-cores of $5 n+4$. We let $P_{t \text {-core }}(m)$ denote the set of $t$-cores of $m$. For $0 \leqslant j \leqslant 4$ we let $P_{t \text {-core }}^{j}(m)$ denote the set of $t$-cores $\tilde{\lambda}$ of $m$, with crank $c_{1}(\tilde{\lambda}) \equiv j(\bmod 5)$. For a $t$-core $\tilde{\lambda}$ we call $\vec{n}=\phi_{2}(\tilde{\lambda})$ its $n$-vector. We define the 5-cycle $\sigma$ in terms of $n$-vectors. The map

$$
\sigma: P_{5-\text { core }}(5 n+4) \longrightarrow P_{5-\text { core }}(5 n+4)
$$


is defined by

$$
\begin{aligned}
\vec{n} \mapsto\left(-\frac{2 n_{0}}{5}+\frac{n_{1}}{5}+\right. & \frac{4 n_{2}}{5}+\frac{2 n_{3}}{5}+\frac{3}{5},-n_{3},-\frac{3 n_{0}}{5}-\frac{6 n_{1}}{5}-\frac{4 n_{2}}{5}-\frac{2 n_{3}}{5}+\frac{2}{5}, \\
& \left.-\frac{n_{0}}{5}+\frac{3 n_{1}}{5}-\frac{3 n_{2}}{5}+\frac{n_{3}}{5}-\frac{1}{5}, \frac{6 n_{0}}{5}+\frac{2 n_{1}}{5}+\frac{3 n_{2}}{5}+\frac{4 n_{3}}{5}-\frac{4}{5}\right) .
\end{aligned}
$$

For each $0 \leqslant j \leqslant 4$, the map

$$
\sigma: P_{5 \text {-core }}^{j}(5 n+4) \longrightarrow P_{5-\text { core }}^{j+1}(5 n+4)
$$

is a bijection.

The key to finding a crank for partitions of $25 n+24$ in [6] was a bijective proof of the identity

$$
a_{5}(5 n+4)=5 a_{5}(n)
$$

The map

$$
\theta: P_{5-\text { core }}(n) \longrightarrow P_{5 \text {-core }}^{0}(5 n+4)
$$

defined by

$$
\begin{aligned}
\vec{n} \mapsto\left(n_{1}+2 n_{2}+2 n_{4}+1,-n_{1}-n_{2}+n_{3}\right. & +n_{4}+1,2 n_{1}+n_{2}+2 n_{3}, \\
& \left.-2 n_{2}-2 n_{3}-n_{4}-1,-2 n_{1}-n_{3}-2 n_{4}-1\right)
\end{aligned}
$$

is a bijection. See [6, p.8]. This together with Theorem 3.2 yields a combinatorial proof of (3.2).

We now describe the crank for 5 -cores of $25 n+24$ found in [6]. For $\lambda \in P_{5 \text {-core }}(25 n+$ 24) choose the unique $\lambda^{\prime} \in P_{5 \text {-core }}^{0}(25 n+24)$ which is in the same orbit as $\lambda$ under the 5-cycle $\sigma$. Define

$$
c_{2}(\lambda):=c_{1}\left(\theta^{-1}\left(\lambda^{\prime}\right)\right)
$$

Let $\vec{n}=\theta^{-1}\left(\lambda^{\prime}\right)$. By $(2.1)$

$$
c_{2}\left(\lambda^{\prime}\right)=c_{1}(\vec{n})=2 n_{1}+n_{2}+2 n_{3} .
$$

Observe that this is the third component in the $n$-vector of $\theta(\vec{n})=\lambda^{\prime}$. It follows that

$$
c_{2}\left(\lambda^{\prime}\right)=r_{2}-r_{3},
$$

where $\vec{r}$ is the $r$-vector of $\lambda^{\prime}$. Unfortunately, it is not true in general that $c_{2}\left(\lambda^{\prime}\right) \equiv c_{2}(\lambda)$ (mod 5). Nonetheless we can find a crank for 5 -cores of $25 n+24$ independent of the two maps $\sigma$ and $\theta$. We have the following 
THEOREM 3.3. Let $\vec{r}=\left(r_{0}, r_{1}, \ldots, r_{4}\right)$ be the $r$-vector of $\lambda$, a 5 -core of $25 n+24$. Then

$$
c(\lambda):=\left(c_{1}(\lambda), c_{2}(\lambda)\right)=\left(2 r_{1}-r_{2}+r_{3}-2 r_{4}, r_{2}-r_{3}\right) \quad(\bmod 5) \in \mathbb{Z}_{5} \times \mathbb{Z}_{5}
$$

is a crank for 5 -cores of $25 n+24$.

Proof: For each $(i, j)$ in $\mathbb{Z}_{5} \times \mathbb{Z}_{5}$, we let $P_{5-\text { core }}^{i, j}(25 n+24)$ be the set of 5 -cores $\lambda$ of $25 n+24$ such that $c(\lambda) \equiv(i, j)(\bmod 5)$. The map

$$
\Psi=\theta \sigma \theta^{-1}: P_{5 \text {-core }}^{0, j}(25 n+24) \longrightarrow P_{5-\text { core }}^{0, j+1}(25 n+24)
$$

is a bijection. We have calculated the effect $\sigma$ has on our crank statistics $c_{1}, c_{2}$. A calculation shows that the map

$$
\sigma: P_{5-\text { core }}^{i, j}(25 n+24) \longrightarrow P_{5-\text { core }}^{i+1, i^{2}+i+j+2}(25 n+24)
$$

is a bijection. We omit the details. We note that the indices are reduced mod 5 . Using the maps $\Psi$ and $\sigma$ we find that

$$
\left|P_{5-\text { core }}^{i, j}(25 n+24)\right|=\left|P_{5-\text { core }}^{0,0}(25 n+24)\right|=\frac{1}{25} a_{5}(25 n+24),
$$

for $0 \leqslant i, j \leqslant 4$. Hence $c=\left(c_{1}, c_{2}\right)(\bmod 5)$ is a crank for 5 -cores of $25 n+24(\bmod 25)$.

A crank for partitions of $25 n+24$ is given in [6, Theorem 6]. This crank is algorithmic in nature. It depends on Bijection 1, and the map $\theta$. In view of Lemma 2.1 and Theorem 3.3, we may define a crank independent of these maps. For a partition $\lambda: \lambda_{1} \geqslant \lambda_{2} \geqslant$ $\cdots \geqslant \lambda_{m}$, with $r$-vector $\vec{r}=\left(r_{0}, \ldots, r_{t-1}\right)$, the definition of $c_{1}(\lambda)$ and $c_{2}(\lambda)$ is analogous to that given for $t$-cores in (3.1), (3.4) respectively. We need two more statistics. We define

$$
s(\lambda):=r_{0}-\left(\sum_{i=0}^{t-1} r_{i}^{2}-r_{i} r_{i+1}\right)
$$

and

$$
c_{3}(\lambda):=\sum_{j=1}^{m}\left(\lambda_{j}-j\right)\left[\frac{\lambda_{j}-j}{t}\right]-\sum_{i=1}^{t-1} i d_{i}\left(\frac{1}{2}\left(d_{i}+1\right)+\left[\frac{m-i-1}{t}\right]\right),
$$

where $d_{i}(\lambda)$ is the number of elements of the sequence

$$
\lambda_{1}-1, \lambda_{2}-2, \ldots, \lambda_{m}-m,
$$

which are congruent to $i(\bmod t)$. Now let $\lambda$ be any partition of $25 n+24$, and suppose

$$
\phi_{1}(\lambda)=\left(\tilde{\lambda}, \hat{\lambda}_{0}, \widehat{\lambda}_{1}, \ldots, \hat{\lambda}_{4}\right) .
$$


Then by Lemma 2.1,

$$
\sum_{i=0}^{4}\left|\widehat{\lambda}_{i}\right|=s(\lambda)
$$

and

$$
\sum_{i=0}^{4} i\left|\widehat{\lambda}_{i}\right| \equiv c_{3}(\lambda) \quad(\bmod 5) .
$$

If $s(\lambda) \equiv 0(\bmod 5)$, then $\tilde{\lambda}$ is a 5 -core with $|\widetilde{\lambda}| \equiv 24(\bmod 25)$ and

$$
c(\lambda):=\left(c_{1}(\lambda), c_{2}(\lambda)\right) \equiv\left(c_{1}(\widetilde{\lambda}), c_{2}(\widetilde{\lambda})\right)(\bmod 5),
$$

since the sum of the coefficients in the definitions of $c_{1}, c_{2}$ is zero. By rewriting [6, Theorem 6] in terms of our new statistics we obtain a bijection independent crank.

THEOREM 3.4. Let $\vec{r}=\left(r_{0}, r_{1}, \ldots, r_{4}\right)$ be the $r$-vector of a partition $\lambda$ of $25 n+24$. We define a crank $c(\lambda) \in \mathbb{Z}_{5} \times \mathbb{Z}_{5}$ as follows.

If $s(\lambda) \equiv 0(\bmod 5)$ we define

$$
c(\lambda):=\left(c_{1}(\lambda), c_{2}(\lambda)\right)=\left(2 r_{1}-r_{2}+r_{3}-2 r_{4}, r_{2}-r_{3}\right) .
$$

If $s(\lambda) \not \equiv 0(\bmod 5)$ we define

$$
c(\lambda):=\left(c_{1}(\lambda), c_{3}(\lambda)\right) .
$$

Then $c(\lambda)$ is a crank for the partitions of $25 n+24 \bmod 25$. The proof utilises Theorem 3.3 and follows from [6, Theorem 6].

3.2. Cranks For partitions of $7 n+5$ AND $49 n+47$ For 7 -cores of $7 n+5$ there is no analog of (3.2) and so there is no analog of the map $\theta$. Nonetheless we are able to find a crank $c(\lambda) \in \mathbb{Z}_{7} \times \mathbb{Z}_{7}$ for the partitions of $49 n+47$.

THEOREM 3.5. [6, p.7] Let $\vec{r}=\left(r_{0}, r_{1}, \ldots, r_{6}\right)$ be the $r$-vector of $\lambda$, a 7-core of $7 n+5$. Then

$$
c_{1}(\lambda):=5 r_{1}-r_{2}-r_{3}+r_{4}+r_{5}-5 r_{6} \quad(\bmod 7) \in \mathbb{Z}_{7}
$$

is a crank for 7 -cores of $7 n+5$.

We make explicit the 7-cycle $\sigma$ that acts on 7 -cores of $7 n+5$. We define the 7 cycle $\sigma$ in terms of $n$-vectors. Since $\sum_{i=0}^{6} n_{i}=0$, we omit the last component $n_{6}$, and let $\vec{n}=\left(n_{0}, n_{1}, \ldots, n_{5}\right)^{T}$.The map

$$
\sigma: P_{7 \text { core }}(7 n+5) \rightarrow P_{7 \text { core }}(7 n+5)
$$

is defined by

$$
\sigma(\vec{n})=M \vec{n}+\vec{\tau}
$$


where

$$
M=\frac{1}{7}\left(\begin{array}{cccccc}
-8 & -2 & -3 & -4 & -5 & -6 \\
1 & 2 & 3 & 4 & -2 & 6 \\
3 & -1 & 2 & 5 & 1 & -3 \\
-2 & -4 & 1 & -1 & 4 & 2 \\
0 & 0 & -7 & 0 & 0 & 0 \\
2 & -3 & -1 & -6 & -4 & -2
\end{array}\right), \quad \vec{\tau}=\frac{1}{7}\left(\begin{array}{c}
5 \\
2 \\
-1 \\
0 \\
-3
\end{array}\right)
$$

We have the following

THEOREM 3.6. Let $\vec{r}=\left(r_{0}, r_{1}, \ldots, r_{6}\right)$ be the $r$-vector of $\lambda$, a 7 -core of $49 n+47$. Then

$$
\begin{aligned}
c(\lambda) & :=\left(c_{1}(\lambda), c_{2}(\lambda)\right) \\
& =\left(5 r_{1}-r_{2}-r_{3}+r_{4}+r_{5}-5 r_{6}, r_{3}+4 r_{4}-4 r_{5}-r_{6}\right) \quad(\bmod 7) \in \mathbb{Z}_{7} \times \mathbb{Z}_{7}
\end{aligned}
$$

is a crank for 7 -cores of $49 n+47$.

PROOF: For each $(i, j)$ in $\mathbb{Z}_{7} \times \mathbb{Z}_{7}$, we let $P_{7 \text {-core }}^{i, j}(49 n+47)$ be the set of 7 -cores $\lambda$ of $49 n+47$ such that $c(\lambda) \equiv(i, j)(\bmod 7)$. We construct 7 bijections

$$
\Psi_{j}: P_{7 \text {-core }}^{0, j}(49 n+47) \longrightarrow P_{7 \text {-core }}^{0, j+1}(49 n+47), \quad 0 \leqslant j \leqslant 6 .
$$

Each map $\Psi_{j}$ has the form

$$
\Psi_{j}(\vec{n})=M_{j} \vec{n}+\vec{\tau}_{j},
$$

where $M_{j}$ is a $6 \times 6$ matrix, and $\vec{\tau}_{j}$ is a constant vector, and which are given below.

$$
\begin{gathered}
M_{0}=\frac{1}{49}\left(\begin{array}{cccccc}
-24 & -36 & -2 & -5 & 13 & -4 \\
40 & 17 & -20 & -1 & 4 & 2 \\
-15 & -37 & -17 & -18 & 23 & -13 \\
0 & 0 & 0 & 49 & 0 & 0 \\
15 & 2 & 38 & -3 & -2 & -22 \\
-40 & -38 & -36 & -41 & -60 & -23
\end{array}\right), \quad \vec{\tau}_{0}=\frac{1}{49}\left(\begin{array}{c}
22 \\
-4 \\
26 \\
0 \\
-26 \\
4
\end{array}\right) \\
M_{1}=\frac{1}{49}\left(\begin{array}{cccccc}
32 & 1 & -30 & -5 & 6 & 10 \\
-9 & -46 & 8 & -15 & 4 & 2 \\
-36 & 12 & -17 & -11 & 16 & 8 \\
0 & 0 & 0 & 49 & 0 & 0 \\
36 & 44 & 52 & 25 & 19 & 48 \\
9 & 11 & 13 & -6 & 17 & -37
\end{array}\right), \quad \vec{\tau}_{1}=\frac{1}{49}\left(\begin{array}{c}
15 \\
31 \\
26 \\
0 \\
-26 \\
-31
\end{array}\right)
\end{gathered}
$$




$$
\begin{aligned}
& M_{2}=\frac{1}{49}\left(\begin{array}{cccccc}
36 & 58 & 24 & 18 & 12 & 27 \\
24 & 6 & 16 & 12 & 57 & 18 \\
-23 & -18 & -48 & -36 & -24 & -54 \\
0 & 0 & 0 & 49 & 0 & 0 \\
23 & -31 & -1 & -13 & -25 & 5 \\
-24 & -6 & 33 & -12 & -8 & -18
\end{array}\right), \quad \vec{\tau}_{2}=\frac{1}{49}\left(\begin{array}{c}
-5 \\
13 \\
10 \\
0 \\
-10 \\
-13
\end{array}\right) \\
& M_{3}=\frac{1}{49}\left(\begin{array}{cccccc}
4 & 8 & -44 & -19 & -8 & -25 \\
-9 & -4 & 22 & -15 & -10 & -40 \\
48 & 54 & 46 & 31 & 37 & 50 \\
0 & 0 & 0 & 49 & 0 & 0 \\
-48 & 2 & -11 & -17 & -2 & 6 \\
9 & -31 & -1 & -6 & 31 & 5
\end{array}\right), \quad \vec{\tau}_{3}=\frac{1}{49}\left(\begin{array}{c}
15 \\
3 \\
-16 \\
0 \\
16 \\
-3
\end{array}\right) \\
& M_{4}=\frac{1}{49}\left(\begin{array}{cccccc}
-12 & 39 & 6 & -6 & -18 & -2 \\
-36 & -30 & 18 & -18 & -5 & -6 \\
31 & -15 & 9 & -9 & -27 & -3 \\
0 & 0 & 0 & 49 & 0 & 0 \\
-31 & -34 & -58 & -40 & -22 & -46 \\
36 & 30 & 31 & 18 & 54 & 6
\end{array}\right), \quad \vec{\tau}_{4}=\frac{1}{49}\left(\begin{array}{c}
11 \\
33 \\
-8 \\
0 \\
8 \\
-33
\end{array}\right) \\
& M_{5}=\frac{1}{49}\left(\begin{array}{cccccc}
-31 & -34 & -58 & -40 & -22 & -46 \\
12 & 10 & -6 & 6 & 18 & 51 \\
-36 & -30 & 18 & -18 & -5 & -6 \\
0 & 0 & 0 & 49 & 0 & 0 \\
36 & 30 & 31 & 18 & 54 & 6 \\
-12 & 39 & 6 & -6 & -18 & -2
\end{array}\right), \quad \vec{\tau}_{5}=\frac{1}{49}\left(\begin{array}{c}
36 \\
24 \\
26 \\
0 \\
-26 \\
-24
\end{array}\right) \\
& M_{6}=\frac{1}{49}\left(\begin{array}{cccccc}
36 & 44 & 52 & 25 & 19 & 48 \\
-32 & -22 & -26 & -37 & -62 & -31 \\
-9 & -46 & 8 & -15 & 4 & 2 \\
0 & 0 & 0 & 49 & 0 & 0 \\
9 & 11 & 13 & -6 & 17 & -37 \\
32 & 1 & -30 & -5 & 6 & 10
\end{array}\right), \quad \vec{\tau}_{6}=\frac{1}{49}\left(\begin{array}{c}
2 \\
20 \\
24 \\
0 \\
-24 \\
-20
\end{array}\right)
\end{aligned}
$$

Let

$$
\begin{aligned}
w(\vec{n}):=w\left(n_{0}, n_{1}, \ldots, n_{5}\right)= & \frac{7}{2}\left(n_{0}^{2}+\cdots+n_{5}^{2}+\left(n_{0}+\cdots+n_{5}\right)^{2}\right) \\
& +n_{1}+2 n_{2}+\cdots+5 n_{5}-6\left(n_{0}+\cdots+n_{5}\right) .
\end{aligned}
$$


In terms of the $n$-vector $c_{1}, c_{2}$ are given by

$$
\begin{aligned}
& c_{1}(\vec{n})=5 n_{1}+4 n_{2}+3 n_{3}+4 n_{4}+5 n_{5}, \\
& c_{2}(\vec{n})=n_{3}+5 n_{4}+n_{5} .
\end{aligned}
$$

In order to show the $\Psi_{j}$ are bijections, we have used computer algebra to show for each $j$,

(i) $\Psi_{j}$ preserves the form $w$,

(ii) $\operatorname{det}\left(M_{j}\right)= \pm 1$, and

(iii) $\vec{n} \in \mathbb{Z}^{6},\left(c_{1}(\vec{n}), c_{2}(\vec{n})\right)=(0, j), w(\vec{n}) \equiv 47(\bmod 49)$ implies $\Psi(\vec{n}) \in \mathbb{Z}^{6}$ and $\left(c_{1}(\vec{n}), c_{2}(\vec{n})\right)=(0, j+1)$.

We have calculated the effect the 7-cycle $\sigma$ has on our crank statistics $c_{1}, c_{2}$. A calculation shows that the map

$$
\sigma: P_{7 \text {-core }}^{i, j}(49 n+47) \longrightarrow P_{7 \text {-core }}^{i+1,4 i+j}(49 n+47)
$$

is a bijection. We omit the details. We note that the indices are reduced mod 7. Using the seven maps $\Psi_{j}$ and the 7-cycle $\sigma$ we find that

$$
\left|P_{7-\text { core }}^{i, j}(49 n+47)\right|=\left|P_{7 \text {-core }}^{0,0}(49 n+47)\right|=\frac{1}{49} a_{7}(49 n+47),
$$

for $0 \leqslant i, j \leqslant 6$. Hence $c=\left(c_{1}, c_{2}\right)(\bmod 7)$ is a crank for 7 -cores of $49 n+47(\bmod 49)$.

Corollary 3.1. Let $\vec{r}=\left(r_{0}, r_{1}, \ldots, r_{6}\right)$ be the $r$-vector of a partition $\lambda$ of $49 n+47$. We define a crank $c(\lambda) \in \mathbb{Z}_{7} \times \mathbb{Z}_{7}$ as follows.

If $s(\lambda) \equiv 0(\bmod 7)$ we define

$$
c(\lambda):=\left(c_{1}(\lambda), c_{2}(\lambda)\right)=\left(5 r_{1}-r_{2}-r_{3}+r_{4}+r_{5}-5 r_{6}, r_{3}+4 r_{4}-4 r_{5}-r_{6}\right) .
$$

If $s(\lambda) \not \equiv 0(\bmod 7)$ we define

$$
c(\lambda):=\left(c_{1}(\lambda), c_{3}(\lambda)\right)
$$

where $c_{3}$ is defined in (3.7).

Then $c(\lambda)$ is a crank for the partitions of $49 n+47 \bmod 49$.

The proof is analogous to that of Theorem 3.4.

\section{REMARKS}

Our cranks for the partitions of $25 n+24$ and $49 n+47$ depend crucially on finding the two crank functions $c_{1}$ and $c_{2}$. The first crank function $c_{1}$ arises naturally from the $t$-cycle one gets from Theorem 3.1. For 5-cores the second crank function $c_{2}$ arises from 
the map $\theta$. We describe another way the second crank function arises. Let $w(\vec{n})$ be defined as in $(3.12)$. Then since $w(\vec{n}) \equiv 5(\bmod 7)$ and assuming $c_{1}(\vec{n}) \equiv 0(\bmod 7)$, there are integers $k, \ell$ such that

$$
\begin{aligned}
& n_{0}=7 k+5-2 n_{1}-3 n_{2}-4 n_{3}-5 n_{4}-6 n_{5} \\
& n_{1}=7 \ell-n_{5}-5 n_{2}-2 n_{3}-5 n_{4} .
\end{aligned}
$$

Now assume the second crank function takes the form

$$
c_{2}(\vec{n})=a b_{2}+n_{3}+b n_{4}+c n_{5},
$$

for some integers $a, b, c$. If we assume $c_{2}(\vec{n}) \equiv 0(\bmod 7)$, then there is an integer $m$ such that

$$
n_{3}=7 m-a n_{2}-b n_{4}-c n_{5} .
$$

We want $w(\vec{n})$ to be a linear form $\bmod 49$ in the remaining variables $n_{2}, n_{4}, n_{5}$. A calculation shows that this can only happen if

$$
(a, b, c) \equiv(0,5,1) \quad(\bmod 7)
$$

which nails down the second crank function $c_{2}$. We have considered the analogous problem for 11-cores of $121 n+116$, and found there is no second crank function of a similar form which makes the corresponding $w(\vec{n})$ linear mod 121 . So if there is a crank for 11-cores of $121 n+116$ it must be more complicated.

It would be interesting to find other occurrences of pairs of crank functions $\left(c_{1}, c_{2}\right)$ which give combinatorial congruences. Zoltan Reti [8] found a pair of crank functions which explains the congruence

$$
s(9 n+8) \equiv 0 \quad(\bmod 9),
$$

where $s(n)$ is the number of partitions of $n$ in which an even part may have two colours. It was Reti's result which led us to search for a function $c_{2}$ for 7 -cores of $49 n+47$.

\section{REFERENCES}

[1] G.E. Andrews, The theory of partitions, Encyclopedia of Mathematics and its Applications 2 (Addison-Wesley Publishing Co., Reading, Mass.-London-Amsterdam, 1976).

[2] G.E. Andrews and F.G. Garvan, 'Dyson's crank of a partition', Bull. Amer. Math. Soc. 18 (1988), 167-171.

[3] A.O.L. Atkin, 'Proof of a conjecture of Ramanujan', Glasgow Math. J. 8 (1967), 14-32.

[4] A.O.L. Atkin and H.P.F. Swinnerton-Dyer, 'Some properties of partitions', Proc. London Math. Soc. (3) 4 (1954), 84-106.

[5] F. Dyson, 'Some guesses in the theory of partitions', Eureka (Cambridge) 8 (1944), 10-15. 
[6] F.G. Garvan, D. Kim and D. Stanton, 'Cranks and t-cores', Invent. Math. 101 (1990), 1-17.

[7] J.G. Kerber, The representation theory of the symmetric group (Addison-Wesley, Reading, MA, 1981).

[8] Z. Reti, Five problems in combinatorial number theory, Ph.D. thesis (University of Florida, 1994).

[9] G.N. Watson, 'Ramanujans Vermutung über Zerfällungsanzahlen', J. Reine. Angew. Math. 179 (1938), 97-128.

Department of Mathematics

University of Florida

Gainesville, FL 32611

United States of America

e-mail: frank@math.ufl.edu 